\title{
Two Jupiter-Mass Planets Orbiting HD 154672 and HD $205739^{1}$
}

\author{
Mercedes López-Morales ${ }^{2,3,4}$, R. Paul Butler ${ }^{2}$, Debra A. Fischer ${ }^{5}$, Dante Minniti ${ }^{6,7}$, Stephen \\ A. Shectman ${ }^{8}$, Genya Takeda ${ }^{9}$, Fred C. Adams ${ }^{10}$, Jason T. Wright ${ }^{11}$, Pamela Arriagada ${ }^{6}$ \\ mercedes@dtm.ciw.edu, fischer@stars.sfsu.edu, paul@dtm.ciw.edu, \\ dante@astro.puc.cl, shec@ociw.edu, genya@u.northwestern.edu, fca@umich.edu, \\ parriaga@astro.puc.cl
}

\begin{abstract}
We report the detection of the first two planets from the N2K Doppler planet search program at the Magellan telescopes. The first planet has a mass of $M \sin i=4.96 M_{\text {Jup }}$ and is orbiting the G3 IV star HD154672 with an orbital period of 163.9 days. The second planet is orbiting the F7 V star HD205739 with an orbital period of 279.8 days and has a mass of $M \sin i=1.37 M_{J u p}$. Both planets are in eccentric orbits, with eccentricities $e=0.61$ and $e=0.27$, respectively. Both stars are metal rich and appear to be chromospherically inactive, based on inspection of their $\mathrm{Ca}$ II H and K lines. Finally, the best Keplerian
\end{abstract}

\footnotetext{
${ }^{1}$ This paper is based on data gathered with the 6.5-meter Magellan Telescopes located at Las Campanas Observatory, Chile.

${ }^{2}$ Carnegie Institution of Washington, Department of Terrestrial Magnetism, 5241 Broad Branch Rd. NW, Washington D.C., 20015, USA

${ }^{3}$ Hubble Fellow

${ }^{4}$ Carnegie Fellow 2004-2007

${ }^{5}$ Department of Physics and Astronomy, San Francisco State University, San Francisco, CA 94132

${ }^{6}$ Department of Astronomy, Pontificia Universidad Católica, Casilla 306, Santiago 22, Chile

${ }^{7}$ Specola Vaticana, V-00120 Citta' del Vaticano Italy

${ }^{8}$ The Observatories of the Carnegie Institution of Washington, 813 Santa Barbara St., Pasadena, CA 91101

${ }^{9}$ Department of Physics and Astronomy, Northwestern University, 2145 Sheridan Road, Evanston, IL 60208

${ }^{10}$ Michigan Center for Theoretical Physics, Physics Department, University of Michigan, Ann Arbor, MI 48109; Astronomy Department, University of Michigan, Ann Arbor, MI 48109

${ }^{11}$ Department of Astronomy, Cornell University, 226 Space Sciences Building, Ithaca, NY 14853
} 
model fit to HD205739b shows a trend of $0.0649 \mathrm{~m} \mathrm{~s}^{-1}$ day ${ }^{-1}$, suggesting the presence of an additional outer body in that system.

Subject headings: Stars: Planetary Systems — Techniques: Radial Velocities — stars: individual (HD 154672, HD 205739)

\section{Introduction}

Ongoing Doppler radial velocity surveys of nearby stars have detected over 200 extrasolar planets in the past decade (Butler et al. 2006). Those surveys focus on late F, G, K, and $\mathrm{M}$ dwarfs within $50 \mathrm{pc}$ and most of the planets they have found to date are more massive than Saturn, and are presumably gas giants. Recently, several Neptune-mass and lower mass planets have been detected, most of them with orbital periods of a few days (Butler et al. 2004; McArthur et al. 2004; Santos et al. 2004; Bonfils et al. 2005, 2007; Rivera et al. 2005; Udry et al. 2006, 2007; Endl et al. 2008)

As the search for new extrasolar planets continues, Doppler surveys now look through a broad parameter space, including long-period Jupiter analogs, very low mass planets in short-period orbits, multiple planetary systems, and new planets around stars with spectral types that extend beyond the ones traditionally been searched, i.e. K0V to F8V. The N2K program (Fischer et al. 2005) is a Doppler survey with distributed observing campaigns at the Keck, Magellan and Subaru telescopes, and is primarily aimed at increasing the number of known hot Jupiters. Because of their proximity to the host stars, the atmospheres of hot Jupiters can be as hot as $2000 \mathrm{~K}$, resulting in detectable emission at IR wavelengths. This makes these short period planets ideal targets for spaceborn follow up to observe exoplanet atmospheres (Harrington et al. 2007), especially when they transit their host stars and undergo a secondary eclipse so that small differential changes in emission from the star-planet system can be measured (e.g. Charbonneau et al. 2005; Knutson et al. 2007). It is also important to have a sample of hot Jupiters that is large enough to provide meaningful constraints on the formation, migration, and evolution mechanisms of these planets (Ford \& Rasio 2006).

The N2K program searches a fresh sample of the "next 2000" stars, not included in other current Doppler surveys, by extending the search radius to distances of 100-110 parsecs from the Sun. This project is intentionally biased towards metal rich stars to exploit the correla-

tion between formation of gas giant planets and high stellar metallicity (Santos et al. 2004; Fischer \& Valenti 2005). The search strategy is optimized for the detection of Jupiter-mass planets with orbital periods shorter than 14 days by obtaining radial velocity measurements 
on three consecutive nights. Variability in the velocity of the host star over this short timescale flags the star as a candidate host for a short-period planet. However, gas giant planets in longer orbits are also identified and detected with additional observations. As an example, the N2K programs at the Keck and Subaru telescopes have already detected seven planets with periods between 21 days and 3.13 years (Fischer et al. 2007; Robinson et al. 2007).

Here, we report the detection of the first two planets from the Magellan N2K program. This program has been underway at the Magellan telescopes at Las Campanas Observatory in Chile since 2004, and includes 300 FGK metal rich stars at distances between 50 and 100 parsecs. The new planets are orbiting the stars HD 154672 and HD 205739. Both host stars showed significant radial velocity scatter in the first year of observations and were initially flagged as hot Jupiter candidates. However, follow-up observations over subsequent years revealed the presence of longer period planets and a trend on the radial velocity curve of HD 205739 that continues to increase after over 3.5 years.

\section{Characteristics of the Host Stars}

HD 154672 is classified as a G3IV star, with apparent magnitude $\mathrm{V}=8.21$ and color B$\mathrm{V}=0.71$ (Hipparcos Catalog; ESA 1997). The Hipparcos parallax of the star is $15.2 \pm 1.11$ milliarcseconds, placing it at a distance of $65.8 \pm 4.8$ parsecs. The distance and the apparent magnitude of the star give an absolute visual magnitude of $M_{V}=4.12$. The bolometric luminosity of the star is $L_{b o l}=1.88 L_{\odot}$, where we have included a bolometric correction of -0.09 derived from the empirical transformations of VandenBerg \& Clem (2003), using the effective temperature, surface gravity and metallicity of the star. Our high resolution spectroscopic analysis, described in Valenti \& Fischer (2005), yields $T_{\text {eff }}=5714 \pm 45 \mathrm{~K}$, $\log \mathrm{g}=4.25 \pm 0.08, v \sin i=1.0 \pm 0.5 \mathrm{~km} \mathrm{~s}^{-1}$, and $[\mathrm{Fe} / \mathrm{H}]=+0.26 \pm 0.04$ for HD154672. The radius of the star derived from the Stefan-Boltzmann relation and the values of the luminosity and effective temperature above is $1.39 R_{\odot}$. We have also derived a stellar mass of $1.06 M_{\odot}$, a radius of $1.27 R_{\odot}$ and an age of about $9.3 \mathrm{Gyr}$ using the Takeda et al. (2007) grid of evolutionary models, based on the Yale Stellar Evolution Code and tuned to the uniform spectroscopic analysis of Valenti \& Fischer (2005). The resultant log $g$ is $4.26_{-0.05}^{+0.06}$, in agreement with the results of the spectroscopic analysis. The uncertainties in these parameters correspond to a $95 \%$ credibility interval using Bayesian posterior probability distributions. The derived stellar parameters of HD154672 are summarized in the second column of Table 1.

The second star, HD 205739, is a F7V with $\mathrm{V}=8.56$ and $\mathrm{B}-\mathrm{V}=0.546$ (Hipparcos 
Catalog; ESA 1997). The Hipparcos parallax of the star is $11.07 \pm 1.12$ milliarcseconds, placing it at a distance of $90.3 \pm 9.1$ parsecs. This sets the absolute magnitude of HD 205739 to $M_{V}=3.78$, and its bolometric luminosity to $L_{b o l}=2.3 L_{\odot}$. The value of $L_{b o l}$, includes a bolometric correction of -0.03 derived from the same empirical transformations of VandenBerg \& Clem (2003) mentioned above. Our spectroscopic analysis yields a $T_{\text {eff }}=$ $6176 \pm 44 \mathrm{~K}, \log \mathrm{g}=4.21 \pm 0.08, v \sin i=4.5 \pm 0.5 \mathrm{~km} \mathrm{~s}^{-1}$, and $[\mathrm{Fe} / \mathrm{H}]=+0.187 \pm 0.05$ Using the effective temperature and stellar luminosity with the Stefan-Boltzmann relation, we calculate the radius of the star to be $1.33 R_{\odot}$. The stellar mass, radius and age derived from the Takeda et al. (2007) grid of evolutionary models are in this case $1.22 M_{\odot}, 1.33 R_{\odot}$, and $2.9 \mathrm{Gyr}$, and $\log \mathrm{g}$ is $4.29_{-0.05}^{+0.06}$. The parameters of HD205739 are summarized in the last column of Table 1.

Finally, the Ca II H \& K lines of HD 154672 and HD205739 (Figure 1) indicate that their chromospheric activity is low. We can therefore reject activity as the cause of the observed radial velocity variations of the stars. Based on these observations, we adopt a conservative upper limit to the expected jitter (or astrophysical noise) of the stars of about $4 \mathrm{~m} \mathrm{~s}^{-1}$ (Wright et al. 2004).

\section{Doppler Observations and Keplerian Fits}

Doppler observations were carried out between mid 2004 and February 2008 at the Magellan Clay telescope using the MIKE spectrograph (Bernstein et al. 2003), with the addition of an iodine cell behind the spectrograph's entrance slit to model the instrumental profile and to set an accurate reference wavelength scale (Butler et al. 1996). The typical signal-to-noise of our spectra is about 130, producing photon-limited uncertainties of 2 to

$4 \mathrm{~m} \mathrm{~s}^{-1}$. Two additional sources of noise are present in the data. The first one is the stellar jitter estimated in $\S 2$. The second source of noise is systematic instrumental errors, which for MIKE has a root mean square (rms) deviation of $5 \mathrm{~m} \mathrm{~s}^{-1}$, as derived from a subset of observed stars that appear to have stable radial velocities over the time span of the observations. A sample of stable stars measured with MIKE is presented in Figures 1 and 2 of Minniti et al. (2008).

We obtained a total of 16 radial velocity measurements for HD 154672 and 24 measurements for HD 205739. Those measurements are summarized in Tables 2 and 3, including the observation dates and the radial velocity formal uncertainties introduced by photon-limited noise. The data are represented in Figures 2 and 3.

For each data set, we modeled the radial velocities to fit single planet Keplerian orbits 
using a Levenberg-Marquardt fitting algorithm. In the case of HD205739 it was necessary to include an additional variable linear trend to best reproduce the observed radial velocity variations. The parameter uncertainties of each best model fit were estimated by running 1000 Monte Carlo trials on each data set, where the model result of each trial was subtracted from the individual data points and the residual velocities scrambled and added back to the velocities predicted by the models, before running a new trial fit. The adopted final uncertainties of each parameter are derived from the standard deviation of all the model trials.

The parameter values of the best Keplerian model fit for each target are summarized in Table 4. The best model for HD154672 has an orbital period of $163.94 \pm 0.01$ days, radial velocity semi-amplitude $K_{1}=225 \pm 2 \mathrm{~m} \mathrm{~s}^{-1}$, and orbital eccentricity $e=0.61 \pm 0.03$. The rms to the fit is $4.36 \mathrm{~m} \mathrm{~s}^{-1}$, with a reduced $\sqrt{\chi_{\sigma}^{2}}=1.60$ relative to the radial velocity formal uncertainties. Adopting a stellar mass of $1.06 M_{\odot}$, we derive a planetary mass of $4.96 M_{J u p}$, and an average relative separation for the system of 0.597 AU. The radial velocity data are plotted in Figure 2, together with the best Keplerian model fit.

In the case of HD 205739, the best model has an orbital period of $279.8 \pm 0.1$ days, radial velocity semi-amplitude $K_{1}=42 \pm 3 \mathrm{~m} \mathrm{~s}^{-1}$, and orbital eccentricity $e=0.27 \pm$ 0.07. The radial velocities also show a substantial linear trend of $0.0649 \pm 0.0002 \mathrm{~m} \mathrm{~s}^{-1}$ per day that continues after over 3.5 years of observations. The rms of the data to the fit is $8.67 \mathrm{~m} \mathrm{~s}^{-1}$, with a reduced $\sqrt{\chi_{\sigma}^{2}}=2.13$ relative to the radial velocity formal uncertainties. Adopting a stellar mass of $1.22 M_{\odot}$, we derive a planetary mass of $1.37 M_{J u p}$, and an average semimajor axis for the system of 0.896 AU. The data with the best Keplerian model fit are represented in Figure 3. As seen in the figure, the residuals to the fit of a single planet plus a long term trend still appear large, showing points that deviate about $2 \sigma$ from the average $\sim 4 \mathrm{~m} \mathrm{~s}^{-1}$ precision of the individual data points, however, an analysis of the periodogram of those residuals reveals no significant peaks, so we can not discard nor confirm the presence of additional shorter period planets in this system with the current dataset.

The amplitude of the observed radial velocity variations for each star is 10-100 times larger than the uncertainties of the individual radial velocity measurements, what makes the possibility that the detected signals are caused by noise fluctuations very unlikely. We quantitatively assert this statement by performing a false alarm probability (FAP) analysis of the data using the method described by Marcy et al. (2005) (see §5.2), with the inclusion of possible linear trends (Wright et al. 2007). Figure 4 shows the result of 1000 FAP trial tests for HD205739. The FAP, i.e. the fraction of trials of scrambled velocities that yields lower $\chi_{\sigma}$ than the best reported fit, is less than $0.1 \%$. A similar analysis of the HD154672 data gives a negligible FAP $(\ll 1.0 \%)$. In this last case, the median $\chi_{\sigma}$ of the FAP histogram 
is about 30 , the first percently $\chi_{\sigma}$ is 16.1 , and the minimum $\chi_{\sigma}$ after 1000 trial tests is 12.5 . None of the FAP trial fits produces a $\chi_{\sigma}$ lower than the value reported above for the best Keplerian fit for HD 154672.

\section{Discussion}

We present two new Jovian-mass planets orbiting metal rich stars.

HD $154672 \mathrm{~b}$ is a fairly massive planet with a mass of Msin $i=4.96 M_{J u p}$ and a very pronounced orbital eccentricity of $e=0.61$, that causes the planet to move from 0.23 to $0.96 \mathrm{AU}$ between periastron and apastron. The planet will therefore experience surface temperature changes of about $300 \mathrm{~K}$ along its orbit, reaching a maximum temperature at periastron of about $600 \mathrm{~K}$, assuming the albedo of the planet is low. If water is present in the atmosphere of HD 154672b, it could transition between gaseous and liquid phases along the planet's orbit.

When placed in the eccentricity vs. orbital period parameter space diagram of known exoplanets illustrated in Figure 5, HD 154672b shows an orbital eccentricity larger than $90 \%$ of the discovered planets, and is only the seventh planet found with an orbital period shorter than 300 days and an eccentricity larger than 0.6. Of the other six planets, four have been found to be either in multiple-planet systems (HD 74156; Naef et al. 2004; Bean et al. 2008), to have a brown dwarf companion (HD 3651; Mugrauer et al. 2006), to be part of a wide stellar binary system (HD 80606; Naef et al. 2001), or to present a large radial velocity trend induced by a distant body associated to that system (HD 37605; Wittenmyer et al. 2007). The high eccentricies in those cases can be explained by either Kozai oscillations (Kozai 1962) or chaotic evolution of planetary orbits in multiple systems. Another planet in this subgroup, HD 17156, has been recently reported to have a large orbital axis misalignment with respect to the stellar rotation axis, that can be best explained by gravitational interactions with other planets (Narita et al. 2007). There is however no evidence for additional objets associated with the last planet in this subgroup, orbiting HD 89744. The radial velocity curve of HD 154672 in Figure 2 shows no trend nor significant residuals to the fit that indicate the presence of other massive objects in that system.

HD 205739b has a mass of Msin $i=1.37 M_{\text {Jup }}$, with an average relative separation of $0.896 \mathrm{AU}$ and an eccentricity of $e=0.27$. In the eccentricity vs. orbital period diagram in Figure 5, the parameters of this planet do not seem atypical. For planets with orbital periods longer than 20 days, the mean eccentricity is 0.29 , therefore HD205739b has an orbital eccentricity that is typical of detected planets that have not experienced tidal circularization. 
The separation of HD205739b from its host star changes from $0.65 \mathrm{AU}$ to $1.14 \mathrm{AU}$ between periastron and apastron. The maximum surface temperature of this planet is expected to be of the order of $400 \mathrm{~K}$, and the amplitude of its surface temperature change will only be of about $100 \mathrm{~K}$ along the entire orbit. One peculiarity of the radial velocity curve of HD 205739 is the presence of a pronounced trend of $0.0649 \mathrm{~m} \mathrm{~s}^{-1}$ per day, indicating the presence of an additional outer body in the system with an orbital period longer than the 3.5 year time span covered by our observations, and a radial velocity semi-amplitude greater than $35 \mathrm{~m}$ $\mathrm{s}^{-1}$. Finally, the residuals of our best fit are a factor of two larger than expected, what hints the possible presence of other bodies in this system. However, more observations are needed to confirm this hypothesis.

We are thankful to the Chilean TAC for awarding extended time to the Magellan N2K project. MLM acknowledges support provided by NASA through Hubble Fellowship grant HF-01210.01-A awarded by the STScI, which is operated by the AURA, Inc., for NASA, under contract NAS5-26555. MLM also acknowledges support from the Carnegie Institution of Washington through a Carnegie Fellowship between 2004-2007, when most of the data presented in this work was collected. RPB has received support from NASA grants NAG5-12182 and NNX07AR40G, NSF grant AST-0307668, and the Carnegie Institution of Washington. DAF acknowledges support from NASA NNG05B164G for the N2K program and support from Research Corporation as a Cottrell Science Fellow. DM and PA are supported by CATA and FONDAP Center for Astrophysics 15010003. FCA has received support for this project from NASA grant NAG5-12182.

\section{REFERENCES}

Bean, J. L., McArthur, B. E., Benedict, G. F., \& Armstrong, A. 2008, ApJ, 672, 1202

Bonfils, X., Forveille, T., Delfosse, X., Udry, S., Mayor, M., Perrier, C., Bouchy, F., Pepe, F., Queloz, D., \& Bertaux, J.-L. 2005, A\&A, 443, L15

Bonfils, X., Mayor, M., Delfosse, X., Forveille, T., Gillon, M., Perrier, C., Udry, S., Bouchy, F., Lovis, C., Pepe, F., Queloz, D., Santos, N. C., \& Bertaux, J.-L. 2007, A\&A, 474, 293

Butler, R. P., Vogt, S. S., Marcy, G. W., Fischer, D. A., Wright, J. T., Henry, G. W., Laughlin, G., \& Lissauer, J. J. 2004, ApJ, 617, 580 
Butler, R. P., Wright, J. T., Marcy, G. W., Fischer, D. A., Vogt, S. S., Tinney, C. G., Jones, H. R. A., Carter, B. D., Johnson, J. A., McCarthy, C., \& Penny, A. J. 2006, ApJ, 646,505

Charbonneau, D., Allen, L. E., Megeath, S. T., Torres, G., Alonso, R., Brown, T. M., Gilliland, R. L., Latham, D. W., Mandushev, G., O’Donovan, F. T., \& Sozzetti, A. 2005, ApJ, 626, 523

Endl, M., Cochran, W. D., Wittenmyer, R. A., \& Boss, A. P. 2008, ApJ, 673, 1165

ESA. 1997, VizieR Online Data Catalog, 1239, 0

Fischer, D. A., Laughlin, G., Butler, P., Marcy, G., Johnson, J., Henry, G., Valenti, J., Vogt, S., Ammons, M., Robinson, S., Spear, G., Strader, J., Driscoll, P., Fuller, A., Johnson, T., Manrao, E., McCarthy, C., Muñoz, M., Tah, K. L., Wright, J., Ida, S., Sato, B., Toyota, E., \& Minniti, D. 2005, ApJ, 620, 481

Fischer, D. A. \& Valenti, J. 2005, ApJ, 622, 1102

Fischer, D. A., Vogt, S. S., Marcy, G. W., Butler, R. P., Sato, B., Henry, G. W., Robinson, S., Laughlin, G., Ida, S., Toyota, E., Omiya, M., Driscoll, P., Takeda, G., Wright, J. T., \& Johnson, J. A. 2007, ApJ, 669, 1336

Ford, E. B. \& Rasio, F. A. 2006, ApJ, 638, L45

Harrington, J., Luszcz, S., Seager, S., Deming, D., \& Richardson, L. J. 2007, Nature, 447, 691

Knutson, H. A., Charbonneau, D., Allen, L. E., Fortney, J. J., Agol, E., Cowan, N. B., Showman, A. P., Cooper, C. S., \& Megeath, S. T. 2007, Nature, 447, 183

Kozai, Y. 1962, AJ, 67, 446

Marcy, G. W., Butler, R. P., Vogt, S. S., Fischer, D. A., Henry, G. W., Laughlin, G., Wright, J. T., \& Johnson, J. A. 2005, ApJ, 619, 570

McArthur, B. E., Endl, M., Cochran, W. D., Benedict, G. F., Fischer, D. A., Marcy, G. W., Butler, R. P., Naef, D., Mayor, M., Queloz, D., Udry, S., \& Harrison, T. E. 2004, ApJ, 614, L81

Minniti, D., Butler, R. P., López-Morales, M., Shectman, S. A., Adams , F. C., Arriagada, P., Boss, A. P., \& Chambers, J. E. 2008, ApJ, submitted

Mugrauer, M., Seifahrt, A., Neuhäuser, R., \& Mazeh, T. 2006, MNRAS, 373, L31 
Naef, D., Latham, D. W., Mayor, M., Mazeh, T., Beuzit, J. L., Drukier, G. A., Perrier-Bellet, C., Queloz, D., Sivan, J. P., Torres, G., Udry, S., \& Zucker, S. 2001, A\&A, 375, L27

Naef, D., Mayor, M., Beuzit, J. L., Perrier, C., Queloz, D., Sivan, J. P., \& Udry, S. 2004, A\&A, 414, 351

Narita, N., Sato, B., Ohshima, O., \& Winn, J. N. 2007, ArXiv e-prints, 712

Rivera, E. J., Lissauer, J. J., Butler, R. P., Marcy, G. W., Vogt, S. S., Fischer, D. A., Brown, T. M., Laughlin, G., \& Henry, G. W. 2005, ApJ, 634, 625

Robinson, S. E., Laughlin, G., Vogt, S. S., Fischer, D. A., Butler, R. P., Marcy, G. W., Henry, G. W., Driscoll, P., Takeda, G., \& Johnson, J. A. 2007, ApJ, 670, 1391

Santos, N. C., Bouchy, F., Mayor, M., Pepe, F., Queloz, D., Udry, S., Lovis, C., Bazot, M., Benz, W., Bertaux, J.-L., Lo Curto, G., Delfosse, X., Mordasini, C., Naef, D., Sivan, J.-P., \& Vauclair, S. 2004, A\&A, 426, L19

Takeda, G., Ford, E. B., Sills, A., Rasio, F. A., Fischer, D. A., \& Valenti, J. A. 2007, ApJS, 168,297

Udry, S., Bonfils, X., Delfosse, X., Forveille, T., Mayor, M., Perrier, C., Bouchy, F., Lovis, C., Pepe, F., Queloz, D., \& Bertaux, J.-L. 2007, A\&A, 469, L43

Udry, S., Mayor, M., Benz, W., Bertaux, J.-L., Bouchy, F., Lovis, C., Mordasini, C., Pepe, F., Queloz, D., \& Sivan, J.-P. 2006, A\&A, 447, 361

Valenti, J. A. \& Fischer, D. A. 2005, ApJS, 159, 141

VandenBerg, D. A. \& Clem, J. L. 2003, AJ, 126, 778

Wittenmyer, R. A., Endl, M., Cochran, W. D., \& Levison, H. F. 2007, AJ, 134, 1276

Wright, J. T., Marcy, G. W., Butler, R. P., \& Vogt, S. S. 2004, ApJS, 152, 261

Wright, J. T., Marcy, G. W., Fischer, D. A., Butler, R. P., Vogt, S. S., Tinney, C. G., Jones, H. R. A., Carter, B. D., Johnson, J. A., McCarthy, C., \& Apps, K. 2007, ApJ, 657, 533

This preprint was prepared with the AAS IATEX macros v5.2. 
Table 1: Stellar Parameters

\begin{tabular}{lcc}
\hline \hline \multicolumn{1}{c}{ Parameter } & HD 154672 & HD 205739 \\
\hline $\mathrm{V} \ldots \ldots \ldots \ldots$ & 8.21 & 8.56 \\
$M_{V} \ldots \ldots \ldots \ldots$ & 4.11 & 3.78 \\
$\mathrm{~B}-\mathrm{V} \ldots \ldots \ldots \ldots$ & 0.71 & 0.54 \\
Spectral Type & $\mathrm{G} 3 \mathrm{IV}$ & $\mathrm{F} 7 \mathrm{~V}$ \\
Distance $(\mathrm{pc})$. & 65.8 & 90.3 \\
$L_{\text {bol }} / L_{\odot} \ldots \ldots$ & 1.88 & 2.3 \\
{$[F e / H] \ldots \ldots \ldots$} & $0.26(0.04)$ & $0.19(0.04)$ \\
$T_{\text {eff }} \ldots \ldots \ldots \ldots$ & $5714(30)$ & $6176(30)$ \\
$v \sin i\left(k m s^{-1}\right)$ & $0.54(0.5)$ & $4.48(0.5)$ \\
$\operatorname{logg}(\mathrm{cgs}) \ldots \ldots$ & $4.25(0.08)$ & $4.21(0.08)$ \\
$M_{\text {star }}\left(M_{\odot}\right)^{\mathrm{a}}$. & $(0.97) 1.06(1.17)$ & $(1.16) 1.22(1.30)$ \\
$R_{\text {star }}\left(R_{\odot}\right)^{\mathrm{a}} \ldots$ & $(1.18) 1.27(1.37)$ & $(1.23) 1.33(1.43)$ \\
Age $(\mathrm{Gyr})^{\mathrm{a}} \ldots$ & $(6.92) 9.28(11.44)$ & $(1.72) 2.84(3.76)$ \\
\hline \hline
\end{tabular}

${ }^{a}$ Values derived from evolutionary models.

Table 2: Radial Velocities for HD 154672

\begin{tabular}{ccc}
\hline $\begin{array}{c}\text { JD-2453000 } \\
(\text { days })\end{array}$ & $\begin{array}{c}\text { RV } \\
\left(\mathrm{ms}^{-1}\right)\end{array}$ & $\begin{array}{c}\sigma_{R V} \\
\left(\mathrm{~ms}^{-1}\right)\end{array}$ \\
\hline 189.7132 & -167.7 & 2.9 \\
190.7083 & -169.7 & 2.8 \\
191.7204 & -173.4 & 3.3 \\
254.5062 & 149.1 & 2.6 \\
596.6893 & 104.9 & 3.1 \\
810.9097 & -31.1 & 2.5 \\
872.8136 & 234.8 & 2.5 \\
1189.8750 & -111.7 & 2.5 \\
1190.8402 & -83.3 & 2.8 \\
1215.8605 & 216.6 & 2.5 \\
1216.7893 & 217.8 & 2.6 \\
1217.8725 & 224.9 & 2.8 \\
1277.7025 & 41.6 & 2.7 \\
1299.6210 & -25.6 & 2.6 \\
1339.5574 & -172.5 & 4.1 \\
1501.8960 & -168.9 & 2.8 \\
\hline
\end{tabular}


Table 3: Radial Velocities for HD 205739

\begin{tabular}{ccc}
\hline \hline $\begin{array}{c}\text { JD-2453000 } \\
(\text { days })\end{array}$ & $\begin{array}{c}\text { RV } \\
\left(\mathrm{ms}^{-1}\right)\end{array}$ & $\begin{array}{c}\sigma_{R V} \\
\left(\mathrm{~ms}^{-1}\right)\end{array}$ \\
\hline 189.8080 & -33.3 & 4.0 \\
190.8643 & -27.7 & 4.4 \\
191.8243 & -31.7 & 4.0 \\
254.6094 & -77.0 & 3.6 \\
550.8993 & -39.0 & 4.3 \\
551.8692 & -57.0 & 3.9 \\
655.6332 & -27.5 & 3.2 \\
657.5999 & -10.4 & 3.6 \\
658.6117 & -11.9 & 3.4 \\
685.5468 & 23.8 & 3.3 \\
872.8832 & -46.8 & 3.5 \\
982.7455 & 38.4 & 4.0 \\
988.7200 & 38.7 & 3.9 \\
1013.6776 & 23.1 & 3.6 \\
1066.5152 & -13.4 & 3.7 \\
1067.5231 & -2.7 & 3.6 \\
1216.9394 & 19.3 & 4.0 \\
1277.8176 & 71.4 & 5.6 \\
1299.8091 & 55.8 & 3.9 \\
1300.7959 & 37.7 & 3.6 \\
1338.7675 & 18.7 & 4.6 \\
1339.7078 & 30.7 & 4.4 \\
1397.5266 & -2.0 & 4.7 \\
1398.5065 & -2.2 & 3.3 \\
\hline
\end{tabular}

Table 4: Orbital Parameters

\begin{tabular}{ccc}
\hline \hline Parameter & HD 154672 & HD 205739 \\
\hline $\mathrm{P}($ days $) \ldots \ldots \ldots \ldots$ & $163.94(0.01)$ & $279.8(0.1)$ \\
$T_{p}(\mathrm{JD}+2450000.) \ldots$ & $3045.3(0.1)$ & $3390.7(0.7)$ \\
$\omega($ deg $) \ldots \ldots \ldots \ldots \ldots$ & $265(2)$ & $301(8)$ \\
Eccentricity $\ldots \ldots \ldots$ & $0.61(0.03)$ & $0.27(0.07)$ \\
$K_{1}\left(\mathrm{~ms}^{-1}\right) \ldots \ldots \ldots \ldots$ & $225(2)$ & $42(3)$ \\
$\mathrm{dv} / \mathrm{dt}\left(\mathrm{ms}^{-1}\right.$ days $\left.^{-1}\right)$ & 0.0000 & $0.0649(0.0002)$ \\
$a_{r e l}(A U) \ldots \ldots \ldots \ldots$ & $(0.580) 0.597(0.617)$ & $(0.881) 0.896(0.915)$ \\
$M \sin i\left(M_{J}\right) \ldots \ldots \ldots$ & $(4.61) 4.96(5.36)$ & $(1.28) 1.37(1.44)$ \\
$N_{\text {obs } \ldots \ldots \ldots \ldots \ldots} \ldots \ldots$ & 16 & 24 \\
$\mathrm{rms}\left(\mathrm{m} s^{-1}\right) \ldots \ldots \ldots$ & 4.36 & 8.67 \\
Jitter $\left(\mathrm{m} s^{-1}\right) \ldots \ldots \ldots$ & 4.0 & 4.5 \\
reduced $\sqrt{\chi_{\sigma}^{2}} \ldots \ldots \ldots$ & 1.60 & 2.13 \\
\hline \hline
\end{tabular}




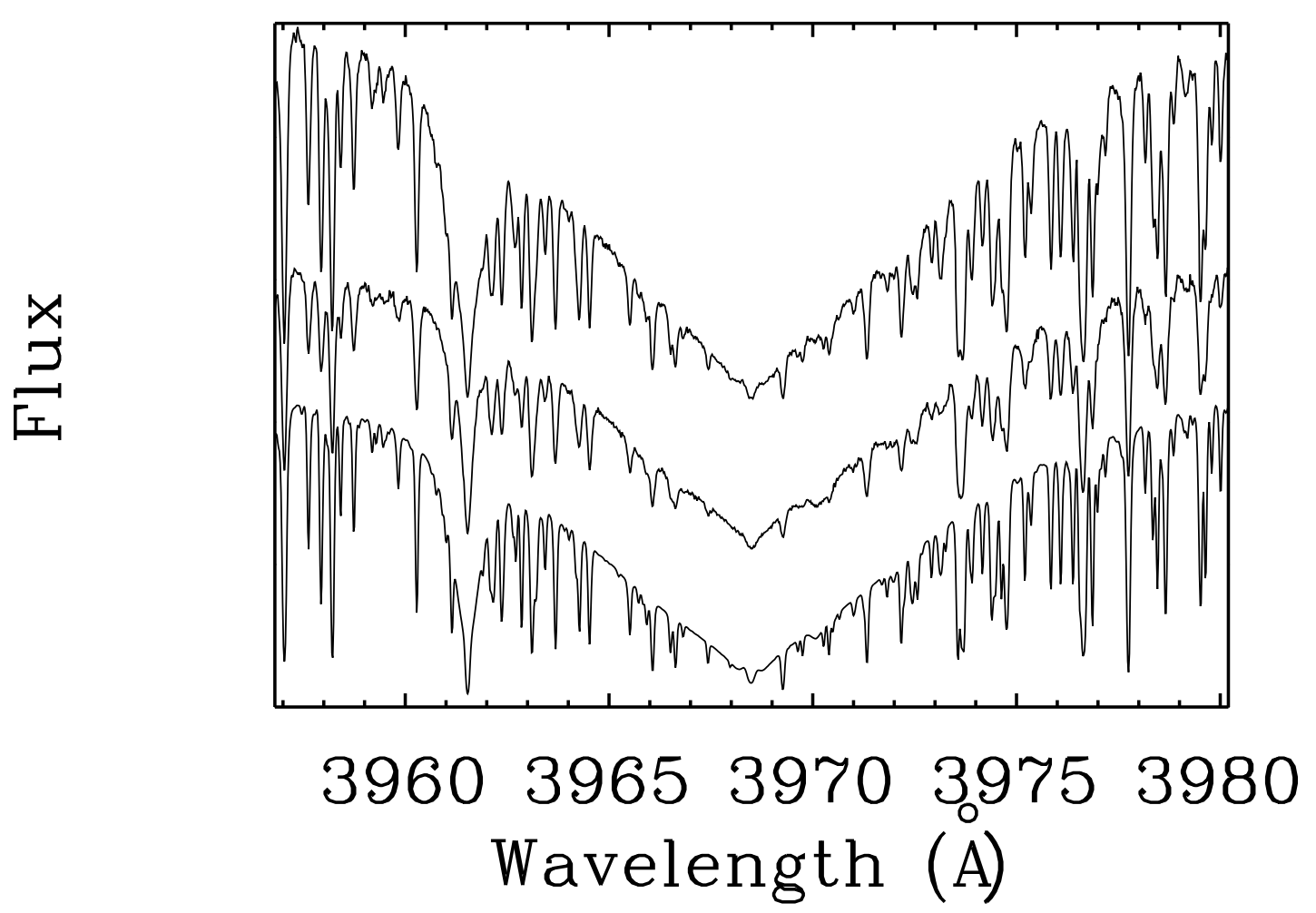

Fig. 1.- CaII H lines of HD 154672 (top) and HD 205739 (middle), compared to the same spectral line region for the Sun (bottom). The lack of emission in the line cores is consistent with low chromospheric activity levels. 


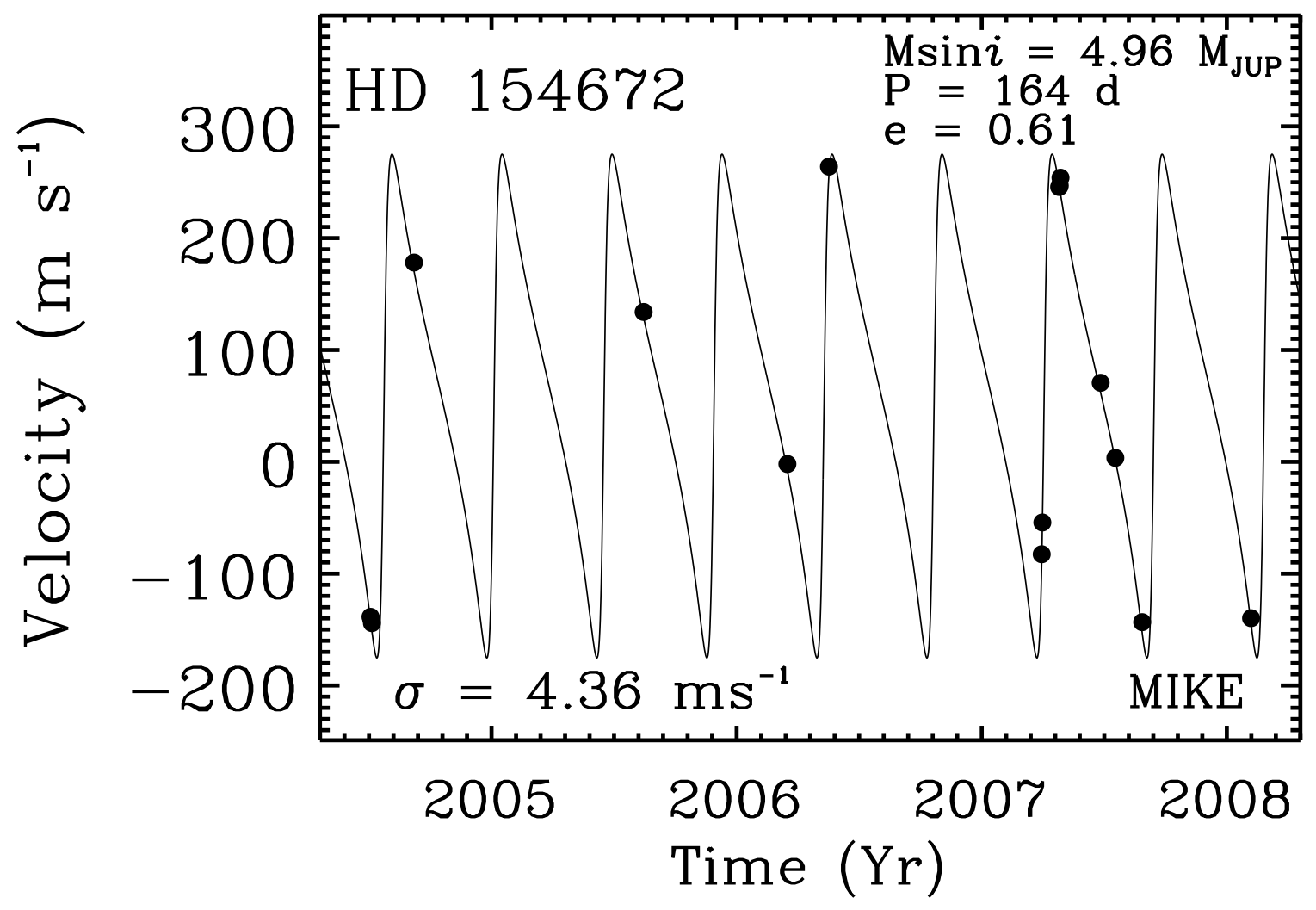

Fig. 2.- Radial velocity measurements for HD154672 over 3.6 years. The minimum mass of the planet, assuming a stellar mass of $1.06 M_{\odot}$, is $M \sin i=4.96 M_{J u p}$. There is no evidence for additional planets in this system. 


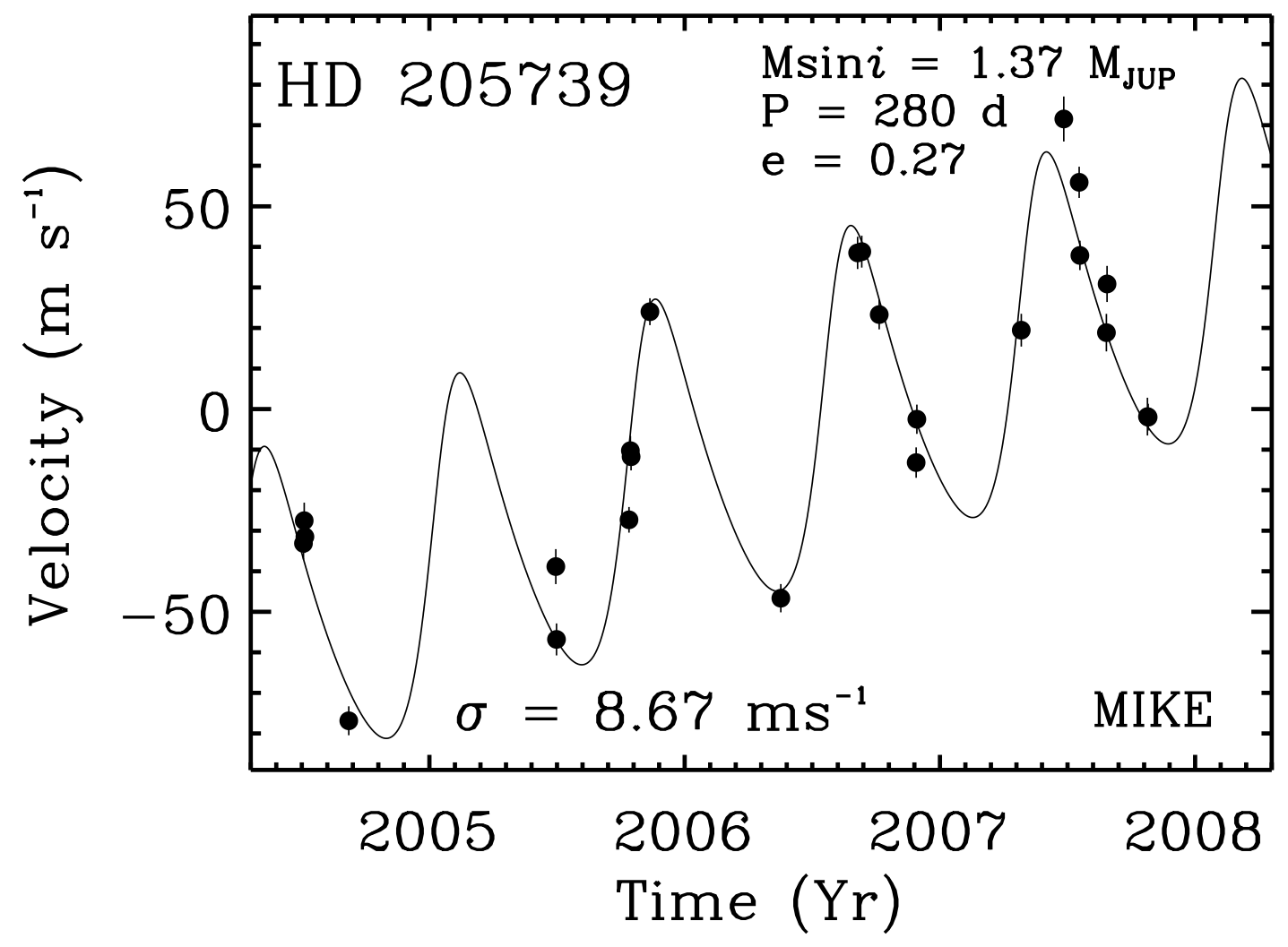

Fig. 3.- Radial velocity measurements for HD205739 over 3.6 years. The minimum mass of the planet, assuming a stellar mass of $1.22 M_{\odot}$, is $M \sin i=1.37 M_{\text {Jup }}$. The best Keplerian fit shows a significant trend of $0.0649 \pm 0.0002 \mathrm{~ms}^{1}$ per day, suggesting the presence of an additional outer body in the system. 


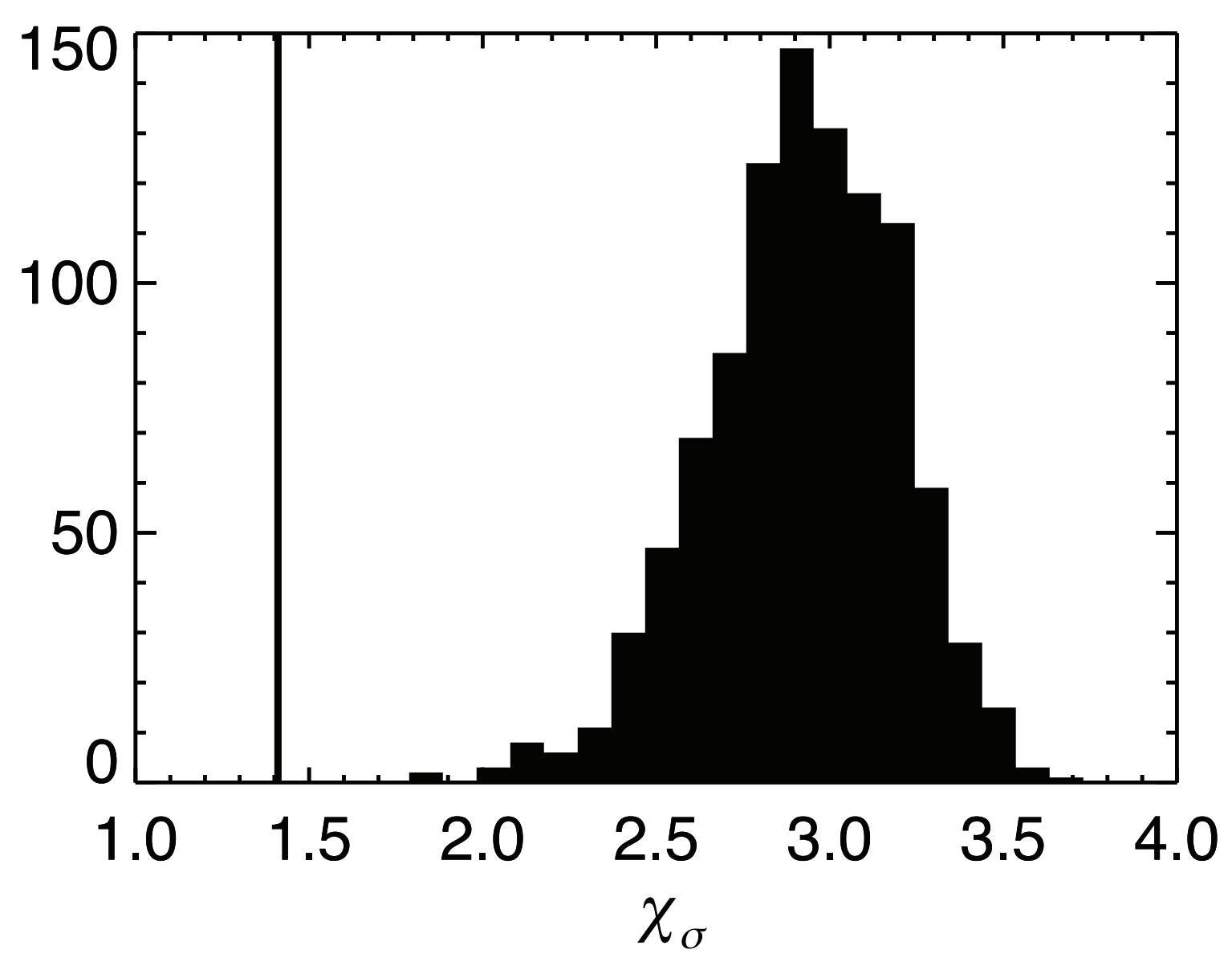

Fig. 4.- Empirical evaluation of the FAP of the single-planet Keplerian fit plus a linear trend model for HD205739 reported in $\S 3$. The $\chi_{\sigma}$ of that fit is $\sim 1.4$ and is indicated by the vertical line in the plot. The vertical axis shows the number of trials that produce a given $\chi_{\sigma}$. Less than 1 of the 1000 trial fits produce $\chi_{\sigma}$ values lower than the original time series of the observations, indicating that the FAP of the reported fit is less than $0.1 \%$. 


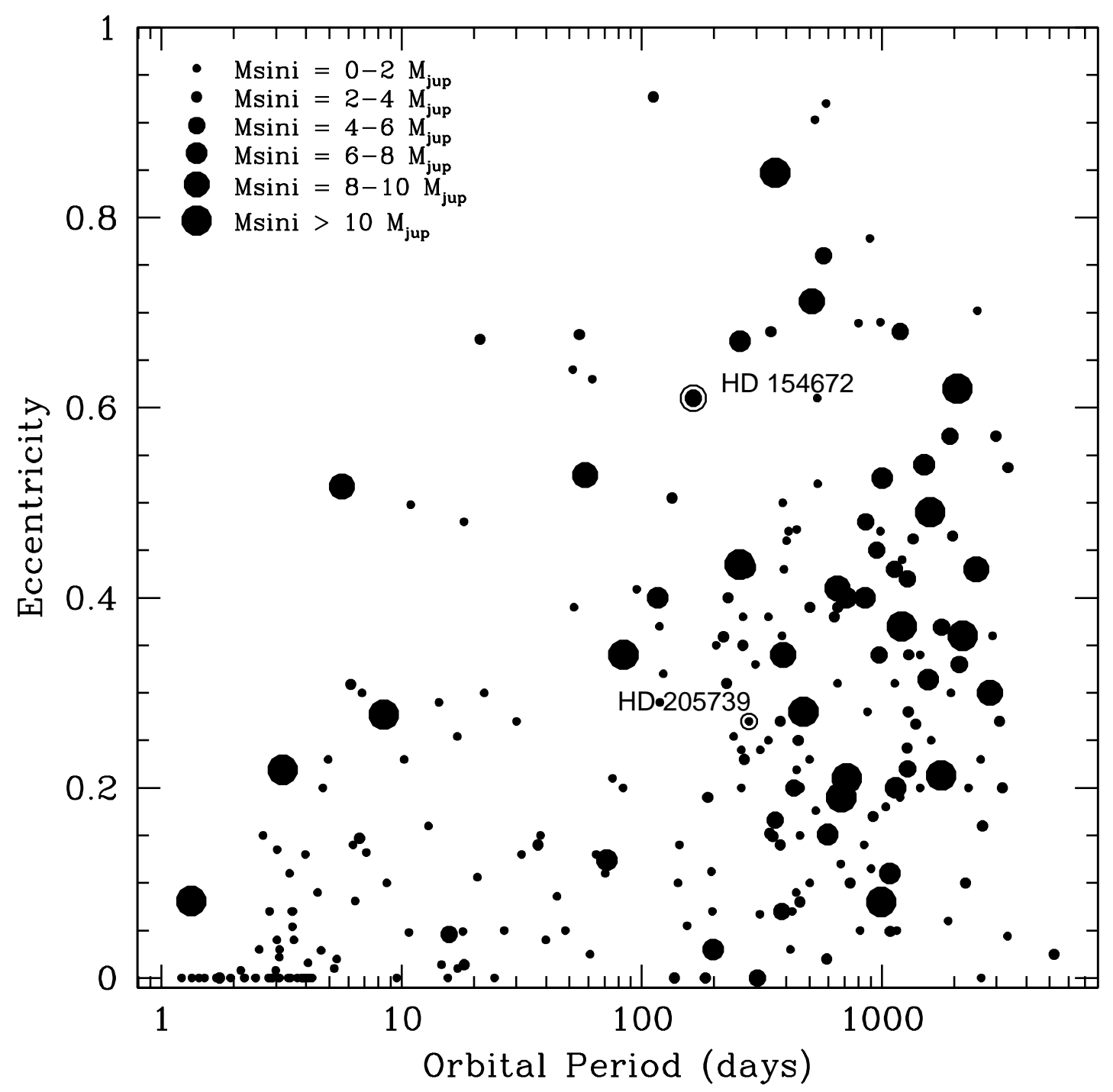

Fig. 5.- Orbital eccentricity versus period diagram for known extrasolar planets. The size of the symbols scales with the planets' mass. The two open circles around the data symbols indicate the location of HD154672 and HD205739 in this diagram. 